\section{MACHINE LEARNING OUTPERFORMS LOGISTIC REGRESSION IN PREDICTING ACCURACY OF CCU ADMISSION FOR HIGH GRADE SEROUS ADVANCED OVARIAN CANCER PATIENTS}

${ }^{1} \mathrm{~A}$ Laios*, ${ }^{2} \mathrm{RV}$ De Oliveira, ${ }^{2} \mathrm{D}$ Lucas, ${ }^{1} \mathrm{Y}$ Tan, ${ }^{1} \mathrm{G}$ Saalmink, ${ }^{1} \mathrm{~A}$ Zubayraeva, ${ }^{1} \mathrm{~A}$ Thangavelu, ${ }^{1} \mathrm{R}$ Hutson, ${ }^{1} \mathrm{~T}$ Broadhead, ${ }^{1} \mathrm{D}$ Nugent, ${ }^{1} \mathrm{G}$ Theophilou, ${ }^{2} \mathrm{KM}$ Gomes de Lima, ${ }^{1} \mathrm{D}$ Dejong. ${ }^{1} \mathrm{St}$ James's University Hospital, Gynaecologic Oncology, Leeds, UK; ${ }^{2}$ Federal University of Rio Grande do Norte, Chemistry, Natal, Brazil

\subsection{6/ijgc-2021-ESG0.304}

Introduction/Background* In advanced stage high grade serous ovarian cancer (HGSOC), the introduction of maximal surgical effort without compromising peri-operative management and subsequent recovery to achieve complete cytoreduction, requires Critical Care Unit (CCU) availability. This paradigm shift prompts the development of tools to accurately predict CCU admission following cytoreductive surgery. Modern data mining technology, such as Machine Learning (ML) could be helpful in accurately predicting CCU admissions to improve standards of care. We developed a framework to improve the accuracy of predicting CCU admission in HGSOC patients by use of ML algorithms (figure 1).

Methodology A cohort of 291 advanced stage HGSOC patients, who underwent surgical cytoreduction from Jan 2014 to Dec 2019, was selected from the ovarian database. They were randomly assigned to training (60\%) and test (40\%) subcohorts. Forward selection and backward stepwise regression were employed to screen independent pre- and intra-operative variables. Linear (LDA), Quadratic (QDA), and non-linear distance (ANN and KNN) ML models were employed to derive predictive information. These methods were tested against conventional linear regression (LR). Model performance was evaluated by prediction accuracy, sensitivity, specificity, and F1 scores.

Result(s)* We identified 56/291(19.2\%) CCU admissions. For the outcome of CCU admission, the prediction accuracies were higher for LDA (0.90) and QDA (0.93) compared with LR (0.84) when all the variables were included in the in-built model. Feature selection identified pre-treatment albumin, surgical complexity score, estimated blood loss, operative time, and bowel resection with stoma formation as the most significant prediction features. With feature selection, the prediction accuracies were higher for LDA (0.89) and KNN (0.86) compared with LR (0.82). Admission to CCU was associated with increased length of stay $(P=0.000)$, and decreased number of postoperative complications $(P=0.001)$.

Conclusion* Herein, ML algorithms accurately predicted HGSOC patients, who required CCU admission following their cytoreductive surgery. Linear discriminant analysis was

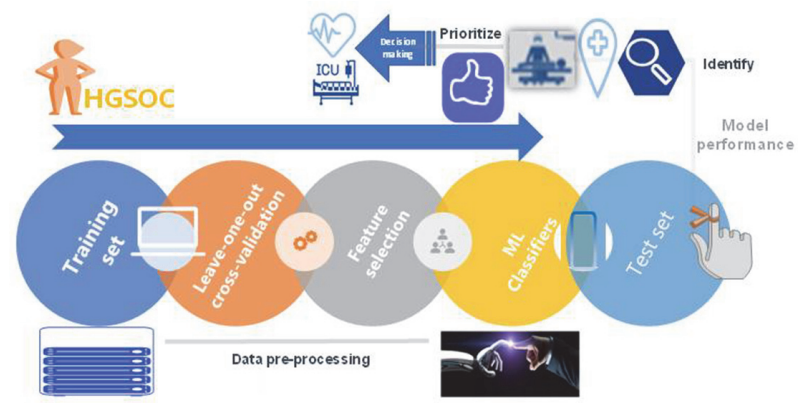

consistently more predictive than LR for CCU admission, irrespective of the number of features included in the analysis. Limited, potentially modifiable, mostly intra-operative factors contributing to CCU admission were identified and suggest areas for targeted interventions.

\section{ERAS PROTOCOLS IN GYNAECOLOGICAL ONCOLOGY. SHOULD WE INCLUDE THORACIC EPIDURAL ANALGESIA?}

${ }^{1} A$ Pandraklakis*, ${ }^{1} A$ Prodromidou, ${ }^{2} \mathrm{~T}$ Lappas, ${ }^{2} \mathrm{E}$ Stamatakis, ${ }^{2} \mathrm{D}$ Valsamidis, ${ }^{1} \mathrm{D}$ Haidopoulos, ${ }^{1} \mathrm{~A}$ Rodolakis, ${ }^{1} \mathrm{~N}$ Thomakos. 'Athens, 1st Department of Obstetrics and Gynecology, Alexandra Hospital, National and Kapodistrian University of Athens, Greece., Athens, Greece; ${ }^{2}$ Department of Anesthesiology and Pain Management, 'Alexandra' General Hospital of Athens, Athens, Greece

\subsection{6/ijgc-2021-ESGO.305}

Introduction/Background* Enhanced recovery after surgery (ERAS) pathways aim to improve the perioperative practice ending in a shorter length of stay with less postoperative complications. Although thoracic epidural analgesia (TEA) is included in ERAS guidelines of other specialties, it is not included in ERAS guidelines in gynaecological oncology. We aim to provide further information in the use of TEA in ERAS protocols in gynaecological oncology.

Methodology A retrospective analysis of a prospectively maintained database of patients who underwent intermediate to high complexity surgery from January 2020 to March 2021 due to gynecological malignancy. The ERAS protocol was followed and patients with compliance rates $>75 \%$ who received postoperative (PO) analgesia through thoracic epidural catheter as part of multimodal analgesia (TEA group) versus those who did not (non-TEA group) were compared. Mobilization, length of stay (LOS), and postoperative pain were considered the primary outcomes of the study

Result(s)* A total of 130 patients (87 in TEA group versus 43 in non-TEA group). Mean visual analog scale (VAS) pain scores at both the day of surgery and PO day 1, were significantly lower in TEA group compared to non-TEA (3.74 vs 4.67, $\mathrm{p}<0.0001$ and 3.30 vs $4.14, \mathrm{p}<0.0001$, respectively). Mobilization rates were significantly higher in TEA group versus non-TEA ( $93 \%$ vs $62 \%, \mathrm{p}<0.0001)$. Mean opioid use was significantly higher in non-TEA group $(p<0.0001)$, while nausea rates were significantly reduced in TEA patients $(\mathrm{p}=0.021)$. No difference in LOS was observed among the two groups (4.18 vs 4.40, $\mathrm{p}=0.995$ ) same as in complication rates.

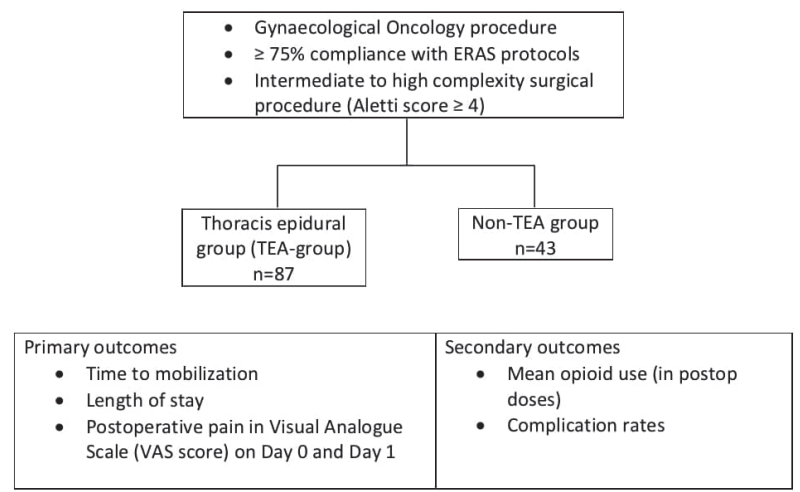

Abstract 474 Figure 1 